\title{
The Direction Cosine Method of Scatterer Location Extended to Spotlight-Mode IFSAR
}

\author{
Paul H. Eichel \\ Sandia National Laboratories
}

\begin{abstract}
A particularly efficient and accurate method of creating digital elevation maps (DEMs) from interferometric SAR data was introduced by the stripmap SAR community. In this paper, we show how to extend this approach, the direction cosine method, to the case of high-resolution spotlight-mode SAR. The method allows the computation of the three dimensional position of scatterers in absolute coordinates directly from the raw radar measurements of range, Doppler, and interferometric phase. Having so computed position triples, the data can then be interpolated onto any map projection desired. The technique is illustrated with actual high-resolution spotlight-mode imagery.
\end{abstract}

\section{Introduction}

An interferometric synthetic aperture radar (IFSAR) uses two or more spatially separated apertures in order to estimate precisely the incidence angle to scatterers on the ground. When combined with the inherent range and azimuth angle measurements of traditional SAR, accurate and detailed 3-D elevation maps of the terrain may be computed in an automated fashion. The stripmapping SAR community was the first to recognize a straightforward and exact method to compute 3-D scatterer locations directly from the three raw radar measurements of range, Doppler, and interferometric phase [1]. In this paper, we extend this approach, the direction cosine method, to high-resolution spotlight-mode SAR. Spotlight-mode SARs permit a longer dwell of the radar on a given patch of terrain allowing the formation of much higher resolution imagery than is possible in stripmap systems [2]. However, the fundamental radar measurements are not exactly the same as for stripmap formed imagery. We show how the stripmap direction cosine method may be modified for the spotlight-mode case. Finally, an example using actual high-resolution spotlight-mode data is provided.

\section{Stripmap-mode IFSAR}

A traditional SAR produces two-dimensional images of the three-dimensional illuminated scene by mapping scatterers into range and Doppler coordinate pairs. Range, as the name implies, is simply the line-of-sight range from the aperture phase center to each given scatterer. Doppler, or range rate, is a function of the angle between the lineof-sight vector and the aperture phase center velocity vector. In a stripmap-mode $\mathrm{SAR}$, the Doppler equation:

$$
f_{D}=\frac{2}{\lambda} \frac{\vec{r}_{l o s} \cdot \vec{v}}{\left|\vec{r}_{l o s}\right|}
$$

relates the (aperture to scatterer) line-of-sight vector, $\bar{r}_{l o s}$, the velocity vector, $\bar{v}$, and the radar wavelength, $\lambda$, to the observed Doppler frequency centroid, $f_{D}[3]$.

At the antenna position corresponding to each radar pulse, a one dimensional array of pixels representing scatterers on the earth with the same Doppler centroid is computed, comprising one column of the two dimensional image. The other dimension (down the column) represents range from that antenna position. These values are computed from pulse return data received over the whole synthetic aperture centered on that position. The aperture is then centered on the next radar pulse position, producing the next column of image data, and so on. Note that this 2D representation of the 3-D world implies geometrical distortion effects such as layover, skew, and superposition.

An interferometric SAR obtains a third measurement value by virtue of a second aperture displaced from the first in cross track. By comparing the phase of the returns as seen by the two apertures, the IFSAR can estimate the incidence angle to scatters in addition to the range and range rate measurements [4]. If we let the vector $\vec{b}$ represent the difference between the aperture phase center positions (the interferometric baseline), then a given scatterer, exhibits a phase difference, $\phi$, given by the relation: 


\section{DISCLAIMER}

This report was prepared as an account of work sponsored by an agency of the United States Government. Neither the United States Government nor any agency thereof, nor any of their employees, make any warranty, express or implied, or assumes any legal liability or responsibility for the accuracy, completeness, or usefulness of any information, apparatus, product, or process disclosed, or represents that its use would not infringe privately owned rights. Reference herein to any specific commercial product, process, or service by trade name, trademark, manufacturer, or otherwise does not necessarily constitute or imply its endorsement, recommendation, or favoring by the United States Government or any agency thereof. The views and opinions of authors expressed herein do not necessarily state or reflect those of the United States Government or any agency thereof. 


\section{DISCLAIMER}

\section{Portions of this document may be illegible in electronic image products. Images are produced from the best available original document.}




$$
\phi=\frac{4 \pi}{\lambda}\left[\frac{\vec{r}_{l o s} \cdot \vec{b}}{\left|\vec{r}_{l o s}\right|}\right]
$$

Thus, the angle between the line-of-sight vector and the baseline can be computed from the phase. (Here, $4 \pi$ is used rather than $2 \pi$, for the case where both antennas are used for transmitting and receiving.) Having a range and two angle measurements for each scatterer, the IFSAR can place those scatters in their true 3-space locations and produce products such as terrain height maps and orthorectified imagery [5].

\section{The direction cosine method}

The direction cosine method ([1]) locates the threedimensional position of scatterers in absolute coordinates directly from the radar measurements of range, Doppler, and interferometric phase. It is computationally efficient, thanks to the selection of a particular orthogonal basis. This basis is chosen with one vector in the direction of the platform velocity vector, a second in the plane defined by the velocity and baseline vectors, orthogonal to the first, and the third to complete a right hand coordinate system:

$$
\begin{aligned}
& \hat{x}=\frac{\vec{v}}{|\vec{v}|} \\
& \hat{y}=\frac{\bar{b}-(\vec{b} \cdot \hat{x}) \hat{x}}{|\vec{b}-(\vec{b} \cdot \hat{x}) \hat{x}|} \\
& \hat{z}=\hat{x} \times \hat{y}
\end{aligned}
$$

The direction cosines of the line-of-sight vector from the radar to a given scatterer with respect to this basis set are simple functions of the radar measurements. We will denote as $\hat{r}=\vec{r}_{\text {los }} /\left|\vec{r}_{\text {los }}\right|^{\text {a unit vector in the line of sight }}$ direction. From Equation (1), we have:

$$
\hat{r}_{x}=\frac{\lambda f_{D}}{2|\vec{v}|}
$$

Similarly, using Equations (2) and (1), we find:

$$
\hat{r}_{y}=\frac{\lambda \phi / 4 \pi-\vec{b}_{x} \hat{r}_{x}}{\vec{b}_{y}}
$$

where

$$
\begin{aligned}
& \vec{b}_{x}=\vec{b} \cdot \hat{x} \\
& \vec{b}_{y}=\vec{b} \cdot \hat{y}
\end{aligned}
$$

are the components of the baseline vector in the $x$ and $y$ basis directions, respectively. Finally, the z-direction cosine is simply.

$$
\hat{r}_{z}= \pm \sqrt{1-\hat{r}_{x}^{2}-\hat{r}_{y}^{2}}
$$

where the sign depends on whether the radar looks left or right of the velocity vector.

Given the line-of-sight unit vector, the range (directly from the pixel row index), and the position of the radar, the scatterer location in absolute coordinates is determined:

$$
\vec{r}_{\text {scauterer }}=\vec{r}_{\text {radar }}+\left|\vec{r}_{\text {los }}\right| \hat{r}
$$

Once the three dimensional position of every scatterer in the image is determined by this procedure, these irregularly spaced triples in 3-space can be interpolated onto any map grid desired.

\section{Spotlight-mode IFSAR}

As previously noted, spotlight-mode SAR permits larger synthetic apertures to be employed by actively steering the antenna to a fixed spot on the ground. These larger apertures, along with wider range bandwidths, result in significantly higher spatial resolution imagery than is typical for stripmap-mode systems. Such radars may also employ a second aperture in an interferometric mode to precisely measure angle of incidence [6]. Among the numerous differences between stripmap and spotlightmode SARs, the measurement of a scatterer's Doppler is the most relevant to the present discussion.

A given pixel in a stripmap SAR image lies at an angle with respect to the velocity vector given by Equation (1). Although it is common practice to adjust the Dopplercentroid of the image processor as a function of slant range, this parameter varies slowly or not at all with pulse number. That is, large blocks of pulses are processed with the same Doppler centroid values as a function of range. Therefore, columns of pixels in the resulting imagery lie along straight lines that are nominally paraillel on_a hypothetical flat earth. See Figure 1.

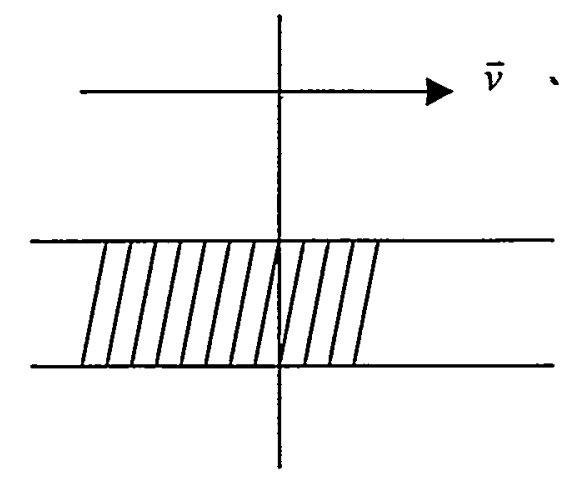

\section{Figure 1. Columns of pixels in a stripmap- mode SAR image.}

In the case of a spotlight-mode SAR, the situation is quite different. All pixels of an entire image are computed from the same synthetic aperture of pulses. For the popular

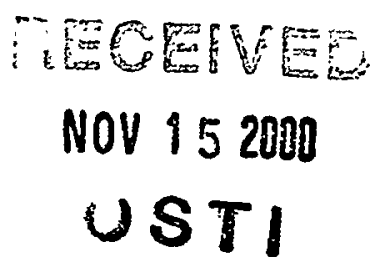


polar formatting algorithm [7], the image pixels end up on a polar range - Doppler sampling grid, but one that is annular in shape rather than rectangular. Thus, pixels comprising a column of the image lie at a fixed angle to the velocity vector at the (common) aperture phase center. See Figure 2.

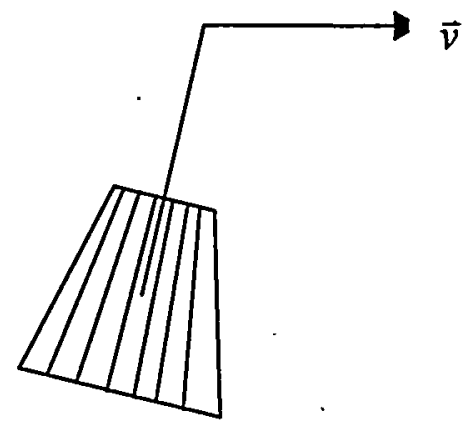

\section{Figure 2. Polar range-Doppler sampling grid for a spotlight-mode image.}

It should be further noted that, prior to image formation, a motion compensation step is typically performed wherein the Doppler of the scene center (or mo-comp point) is removed from the entire phase history.

In such an image, every pixel in a given column has the same true Doppler (i.e. angle off of the velocity vector). This may be computed by finding the relative Doppler of that column (a function only of its column distance from scene center), and adding in the Doppler of the mo-comp point removed by motion compensation. Mathematically, we again have:

$$
\hat{r} \cdot \vec{v}=\frac{\lambda f_{d}}{2|\vec{v}|}
$$

but this time, each column of pixels has a different Doppler given by:

$$
f_{d}(k)=\left(k-k_{m c p}\right) f_{s s}+f_{m c p}
$$

where $\mathrm{k}$ is the column index, $k_{m c p}$ is the column containing the mo-comp point, $f_{s s}$ is the Doppler sample spacing in azimuth used by the processor, and $f_{m c p}$ is the Doppler to the mo-comp point. Note also that the aperture phase center (at which point the Doppler angle is measured) is common for all pixels of the image, not moving from column to column as in the stripmap case. Range is simply a function of the row index of each pixel just as in the stripmap case. Likewise, the angle between the line of sight and baseline vectors is calculated from the unwrapped interferometric phase using Equation (2).

Given the above, the modifications to the direction cosine method for spotlight-mode IFSAR are modest. First, the line of sight vectors to each scatter are computed relative to a single aperture phase center, rather than one that moves along track pulse by pulse. This single aperture phase center becomes $\vec{r}_{\text {radar }}$ in Equation (8) for every scatterer in the image. Second, the direction cosine of $\vec{r}_{\text {los }}$ with respect to the $\mathrm{x}$ basis vector is still given by Equation (4). However, the computed Doppler of Equation (10) replaces the Doppler centroid in that equation. The basis vectors themselves are determined by the velocity and baseline vectors in identical fashion to the stripmap-mode case.

\section{A Spotlight-mode IFSAR example}

The procedure is demonstrated with some actual high resolution spotlight-mode SAR imagery collected by the RTV (Rapid Terrain Visualization) platform [8]. Numerous $1.5 \mathrm{~km} \mathrm{X} 0.3 \mathrm{~km}$ patches of terrain are collected and mapped by the procedure to a UTM map projection. The individual but overlapping patches are then mosaicked together to produce the final output product. Figure 3 . shows a portion of a $7.5 \times 7.5$ minute DEM produced with a $10 \mathrm{~m}$ post spacing. The raw radar imagery was produced at approximately $1 \mathrm{~m}$ resolution, thus providing many looks per output post. At this resolution, streets comprising housing subdivisions are easily distinguished from the houses themselves on the basis of height.

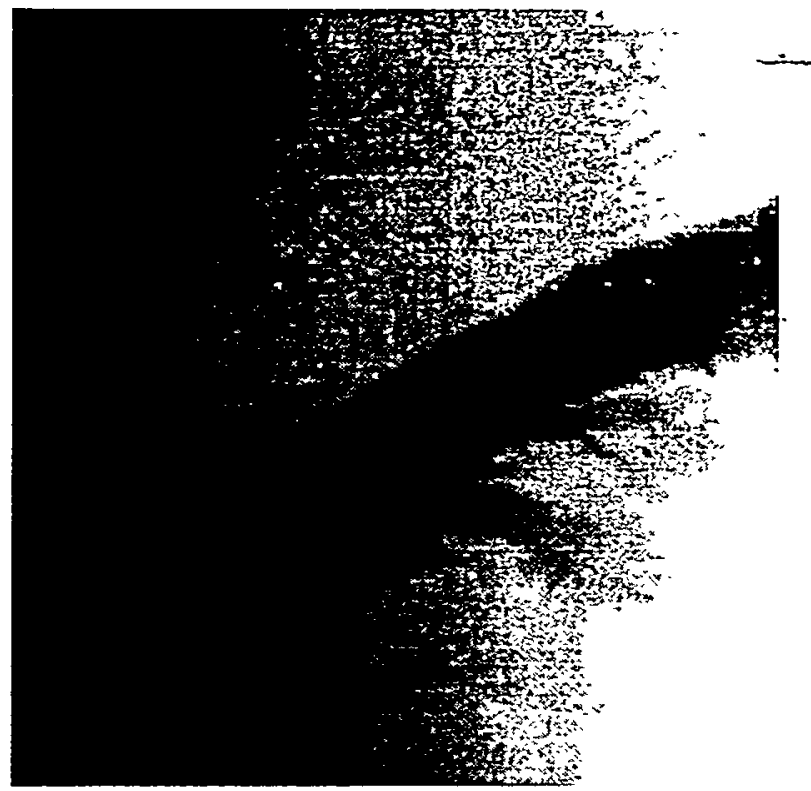

Figure 3. A DEM produced on a $10 \mathrm{~m}$ post spacing from $1 \mathrm{~m}$ resolution spotlightmode SAR imagery. 
The orthorectified imagery corresponding to this DEM is shown in Figure 4. This image is produced at a $2.5 \mathrm{~m}$ post spacing.

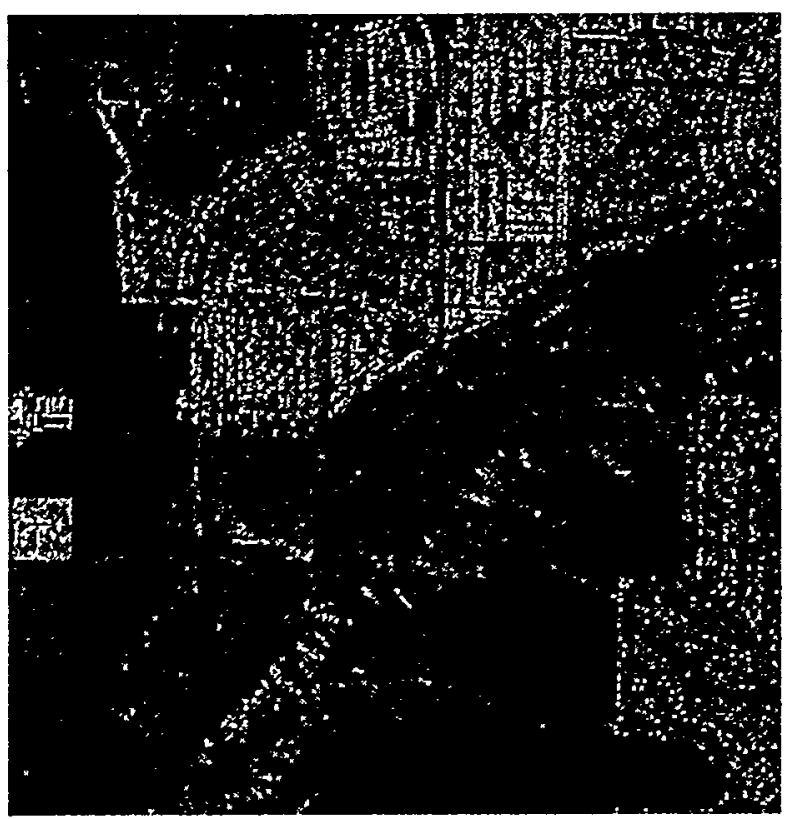

Figure 4. Orthorectified image corresponding to DEM in Figure 3.

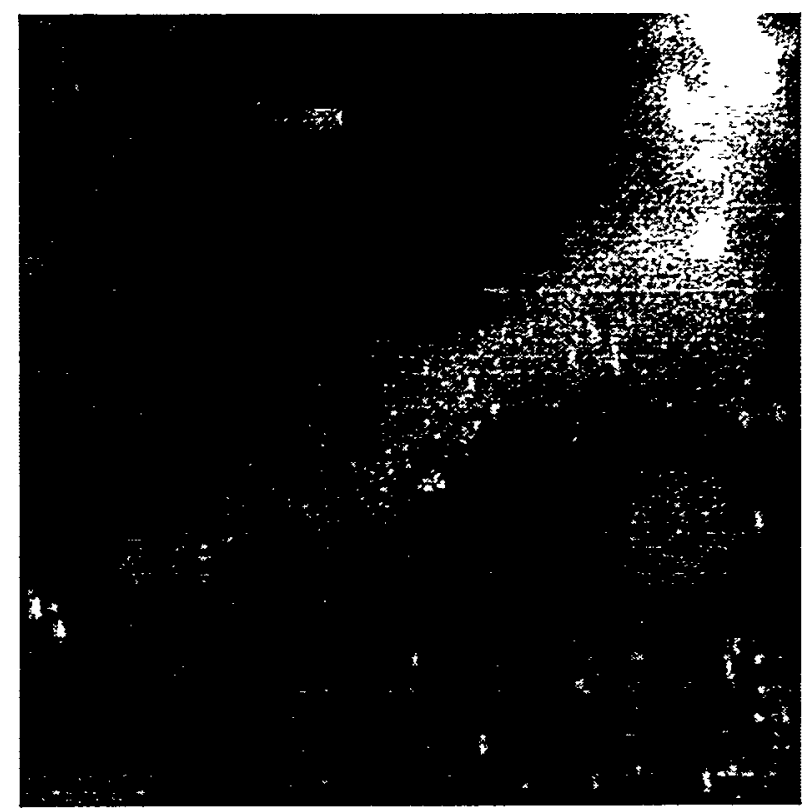

Figure 5. A DEM produced on a $3 \mathrm{~m}$ post spacing from $0.4 \mathrm{~m}$ resolution spotlightmode SAR imagery.

A higher resolution DEM is shown in Figure 5. This DEM is part of a $4.0 \times 4.0$ minute map produced from
$0.4 \mathrm{~m}$ resolution imagery and is computed on a $3 \mathrm{~m}$ post spacing. At this resolution, individual houses are easily recognized. In the lower right of this image, houses comprising a small subdivision of the town of Twin Lakes, NM can be seen. The structure at the top left can be seen to be one story tall in the west half and two stories tall in the east half. Some roads may be distinguished in the DEM by virtue of the fact that they have been filled above grade.

\section{Conclusions}

In this paper we have shown how the direction cosine method of stripmap-mode IFSAR may be modified for use in the spotlight-mode case. Spotlight-mode IFSAR geometry dictates a common aperture phase center, velocity vector, and baseline vector for every pixel in an image. Angle with respect to the velocity vector is the same for every pixel in a given column and can be computed from the column index, the Doppler of the motion compensation point, and the Doppler column sample spacing used in image formation. With these modifications, the direction cosines and length of the line of sight vector to every scatterer in the scene may be computed directly from the raw radar measurements of range, Doppler, and interferometric phase.

\section{References}

[1] S. N. Madsen, H. A. Zebker, and J. Martin, "Topographic mapping using radar interferometry: processing techniques, IEEE Trans. Geoscience and Remote Sensing, Vol. 31, No. 1, pp. 246-256, 1993.

[2] W. G. Carrara, R. S. Goodman, and R. M. Majewski, Spotlight Synthetic Aperture Radar: Signal Processing Algorithms, Artech House, p. 5, 1995.

[3] J. C. Curlander and R. N. McDonough, Synthetic Aperture Radar; Systems and Signal Processing, Wiley, p. 168, 1991. [4] L. C. Graham, "Synthetic Interferometer Radar for Topographic Mapping," Proc. IEEE, vol. 62, pp. 763-768, 1974.

[5] F. W. Leberl, Radargrammetric Image Processing, Artech House, pp. 206-212, 1990.

[6] C. V. Jakowatz, Jr., et. al., Spotlight-Mode Synthetic Aperture Radar: A Signal Processing Approach, Kluwer, pp. 303-308, 1996.

[7] D. A. Ausherman, et. al., "Developments in Radar Imaging," Proc. IEEE, vol. 72, pp. 661-669, 1984.

[8] B.L. Burns, P.H. Eichel, W.H. Hensley, and T.J. Kim, "IFSAR for the Rapid Terrain Visualization Demonstration," Proc. 34 ${ }^{\text {th }}$ Asilomar Conf. on Sig, Sys, \& Comp, Oct. 2000.

Sandia is a multiprogram laboratory operated by Sandia Corporation, a Lockheed Martin Company, for the United States Department of Energy under Contract DE-AC0494AL85000. 